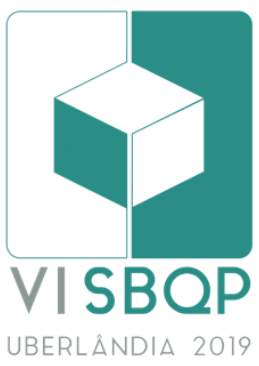

\title{
A CIRCULAÇÃO EM ARQUITETURA: COM BASE EM ANÁLISE DE PROJETO
}

\author{
VICTORIO, Evandra R. \\ FEC Unicamp, evandra@robertoleme.com \\ KOWALTOWSKI, Doris C. C. K. \\ FEC Unicamp, doris@fec.unicamp.br
}

\begin{abstract}
RESUMO
A presente pesquisa de mestrado, já finalizada, tratou da questão da circulação de pessoas e bens no espaço físico arquitetônico. Essas questões, geralmente subestimadas nas fases de análise do processo projetual, impactam a configuração do espaço, sua articulação e funcionalidade. Como o processo de projeto em arquitetura, na contemporaneidade, tornou-se mais complexo, aumentaram também as responsabilidades sobre o profissional e a necessidade de ferramentas de apoio com base em conhecimento científico e análise de projetos. Assim, um conjunto de parâmetros capazes de apoiar o processo de projeto foi levantado na pesquisa, por meio de análise da arquitetura contemporânea. A metodologia consistiu na análise gráfica do papel da circulação em exemplos de projetos arquitetônicos contemporâneos. A amostra foi constituída de quatro projetos de biblioteca - com informações técnicas de suporte -, laureados com o prêmio Pritzker de Arquitetura entre 2000 e 2017. Os resultados foram metodológicos e conceituais, com a criação de uma matriz gráfica de conceitos das questões de circulação em arquitetura. $\bigcirc$ método de análise gráfica demonstrou-se eficiente para o levantamento de novos conceitos sobre circulação em arquitetura, e essa nova matriz foi considerada importante contribuição na prática do processo de projeto profissional e acadêmico, por permitir resolução e configuração mais criativas para o espaço arquitetônico.
\end{abstract}

Palavras-chave: processo de projeto; circulação em arquitetura; biblioteca; análise gráfica de projeto.

\begin{abstract}
The present master's research, already finished, investigated the role of circulation of people and goods in architecture. The questions of circulation, usually underestimated in the analysis phase of the design process, impact the configuration of space, its articulation and functionality. As the design process in architecture, in the contemporaneity, became more complex, also increased the responsibilities on the professional and the need of support tools based on scientific knowledge and analysis of projects. Thus, a set of parameters able to support the design process was created in the research, through analysis of contemporary architecture. The methodology consisted of the graphic analysis of the role of circulation in examples of contemporary architectural projects. The sample consisted of four library projects - with technical support information - of architects awarded the Pritzker Prize between 2000 and 2017. The results were methodological and conceptual, with the creation of a graphic matrix of concepts of circulations issues in architecture. The graphical analysis method proved to be efficient in revealing new concepts about circulation in architecture, and this new matrix was considered an important contribution in the practice of the professional and academic design process, because it allows more creative resolution and configuration for the architectural space.
\end{abstract}

Keywords: Design process; Circulation in architecture; Library; Graphic design analysis.

VICTORIO, E. R.; KOWALTOWSKI, D. C. C. K. A circulação em arquitetura: com base em análise de projeto. In: SIMPÓSIO BRASILEIRO DE QUALIDADE DO PROJETO NO AMBIENTE CONSTRUÍDO, 6., 2019, Uberlândia. Anais... Uberlândia: PPGAU/FAUED/UFU, 2019. p. 375-387. DOI https://doi.org/10.14393/sbqp19036. 


\section{INTRODUÇÃO}

O processo de concepção de projeto em arquitetura e a configuração de novas espacialidades foram ampliados com a recente incorporação do conceito contemporâneo de fluidez à Arquitetura (ROCHA, 2015). Esse novo componente no processo de projeto levou ao surgimento de preocupações da área quanto às questões de circulação em arquitetura. A literatura sobre o recorte "circulação", como um elemento de síntese no projeto de arquitetura, é ainda escassa. Porém trata-se de uma questão presente, ainda que de forma não explícita, nas discussões sobre o processo de projeto (VAN DER VOORDT; VAN WEGEN, 2013). Dessa forma os conceitos que compõem a circulação (orientabilidade, hierarquização, organização etc.) foram investigados nesta pesquisa por meio da análise gráfica de projetos referenciais, desenvolvidos no período atual, os quais devem contribuir para o conhecimento das características das respectivas edificações e para respostas mais criativas na resolução de problemas de fluxo na Arquitetura.

A questão que permeia o estudo é a de que a circulação define a configuração do espaço arquitetônico por meio da articulação do programa de necessidades. Porém, nas reflexões que compõem as fases de análise e síntese do processo projetual, comumente as questões de circulação são subestimadas em sua complexidade. Como consequência, na sua maioria dos projetos, os espaços destinados à circulação não fazem parte de programas de necessidades. E verifica-se, ainda, uma dificuldade na descrição dos impactos da circulação sobre o projeto.

Dessa forma, esta pesquisa teve, como objetivo geral, levantar, a partir do conceito de circulação em arquitetura, um conjunto de parâmetros, abordagens, elementos organizadores e soluções de problemas capazes de apoiar o processo de projeto. Para alcançar esse objetivo, foi ainda objetivo específico: estudar o papel da circulação (fluxo) de pessoas no projeto de arquitetura a partir de elementos de análise gráfica e sua representação. Justifica-se a pesquisa na medida em que os resultados apoiam o processo de projeto com a estrutura de uma matriz gráfica de soluções de problemas de circulação em arquitetura.

\subsection{Circulação no projeto arquitetônico}

A arquitetura em si é um objeto físico, estático, compreendido pelo movimento (circulação) incorporado pela ação humana, e o projeto arquitetônico da contemporaneidade deve ser capaz de ordenar esse movimento e sua duração, a fim de atender às várias demandas da sociedade, inclusive à do fluxo da informação (SOLA-MORALES, 2002).

Dentre os fatores que interferem no processo de concepção do espaço como as variáveis funcionais, volumétricas, espaciais, ambientais, de custos, de desempenho e tecnológicas -, a circulação é a base de decisões estratégicas do projeto, por ser articuladora de funções, definidora de formas e volumes, além de provedora de sensações, percepções e orientabilidade [wayfinding]'. Ela está associada à otimização das atividades definidas no

\footnotetext{
1 A orientabilidade está relacionado à compreensão do espaço e das relações entre objetos e lugares, e depende da habilidade cognitiva e comportamental. O "navegar" por um espaço representa a composição entre o wayfinding - elemento cognitivo que envolve a capacidade de perceber, compreender e memorizar um espaço e/ou um percurso, ou seja, trata-se da
} 
programa de necessidades para determinado espaço (VAN DER VOORDT; VAN WEGEN, 2013) e também à função econômica, principalmente pela área proporcional do projeto destinada ao seu uso. Quanto ao posicionamento e perfil, a circulação pode ter o propósito de resolver questões de conforto; ser referência estrutural e visual do projeto; ser portadora de significados simbólicos e culturais; e ser tratada como uma questão estética do projeto.

Peña e Parshall (2012) definem as questões da circulação por meio de conceitos como: hierarquia, acessibilidade, sistemas de fluxos de pessoas, de veículos e de mercadorias, orientação, flexibilidade e segurança. Esses autores contemplam tais fatores no programa de necessidades. Já os conceitos utilizados por Clark e Pause (1996) são os de composição e estruturação arquitetônica (funcional, construtiva, estética, compositiva, de volumetria, adição e subtração) para unificar e categorizar aspectos de arquitetura em um mesmo padrão de representação. Desses elementos, o aspecto da circulação para a área útil [circulation to use-space] considera a representação dos elementos estáticos (área útil) e dinâmicos (circulação) como essenciais ao projeto. A área útil é o centro das principais decisões projetuais tomadas em relação à função e pode corresponder a áreas abertas ou fechadas.

Ching (2008), por sua vez, parte do estudo dos elementos da forma, por meio da análise de soluções apresentadas em projetos já existentes, para entender as organizações espaciais. Ele define os conceitos de sistema, ordem, forma e espaço, e a circulação compõe os sistemas arquitetônicos. Ela é discutida como elemento de movimento no espaço e no tempo, com abordagem: da aproximação e entrada; configuração do caminho e acesso; sequência de espaço; influência da luz; levando em conta também aspectos relativos ao usuário, tais como visão, audição, olfato e tato.

Já Unwin (1997) entende que a definição das estratégias de organização do espaço é fundamental e traduzida pela estratificação e transição, assim como pela hierarquia e pelo núcleo - que se referem à relação entre lugares estáticos e lugares dinâmicos (circulação) -. Os lugares dinâmicos e de transição são fundamentais para a organização conceitual do espaço e determinantes para a estrutura hierárquica do espaço. Baker (1998), em contrapartida, afirma que a arquitetura está condicionada ao lugar, aos requisitos funcionais e à cultura do local. Como em um sistema, em que as várias partes são organizadas em relação a uma ideia temática. Em um projeto de arquitetura, segundo este autor, a circulação é considerada determinante para a criação de rotas, e, como estas têm energia própria, em arquitetura elas são percebidas como forças. O movimento é classificado pelo autor como um elemento importante de projeto, em que escadas, rampas e elevadores adquirem forças de diferentes intensidades. Cada tipo de movimento possui características particulares e uma relação potencial com seu entorno imediato, que o distingue do resto.

representação mental de um ambiente - e o movimento, como elemento motor (DARKEN; PETERSON, 2001). O wayfinding arquitetônico tem o foco no ambiente construído e no ambiente urbano, e seus aspectos estão relacionados à legibilidade do espaço e de seus limites; à articulação e identificação clara dos espaços; ao zoneamento coerente com a função e/ou destino; ao sistema de circulação legível e com comunicação integrada; à capacidade de proporcionar experiências espaciais agradáveis, seguras e acessíveis (HUNTER, 2010). 
A organização espacial dos edifícios influencia a maneira como as pessoas os utilizam (NATAPOV; DALTON; HÖLSCHER, 2015), e essa abordagem está baseada nas características do comportamento humano de comunicação, movimentação e orientação. Por isso, o sistema de circulação (corredores, conexões verticais, saguões) é determinante para os padrões de movimento das edificações, e entender essa questão pode permitir ao arquiteto contribuir para soluções espaciais que facilitem o acesso, estimulem a circulação natural e a identidade cultural do espaço.

\subsection{Arquitetura fluída}

No processo de industrialização do século XX, o termo "aparência atraente" (beleza), perdeu importância à medida que a "linguística estrutural" passou a dominar o discurso formal do arquiteto. $O$ interesse pela estabilidade estrutural também se enfraqueceu desde os anos 1960, quando houve uma redução progressiva da massa volumétrica das construções. Essa diminuição, ao longo das décadas, deu condições para que os arquitetos pudessem "compor, decompor e recompor campos volumétricos de forma arbitrária, guiados por leis formais e não mais estruturais" (TSCHUMI, 2008, p. 180).

Essa nova estrutura de espaço arquitetônico - descontínua, relativa e heterogênea - é conflitante com sua dimensão substancial - homogênea e contínua -, herdadas dos conceitos clássicos de geometria (VIRILIO, 1993). Por outro lado, a cultura contemporânea, vinculada aos fluxos, prioriza a transformação e os processos estabelecidos e modificados pelo tempo. Nesse cenário, formalmente a arquitetura deve ter características adequadas para incorporar a mudança e dar forma física a uma experiência de durabilidade em tal mudança, algo bem diferente do desafio do tempo, caracterizado pelo pensar clássico.

Tal transição entre os conceitos vitruvianos, de uma arquitetura sólida, para a estrutura de uma arquitetura líquida e fluida, da contemporaneidade, pode ser definida a partir das condicionantes materiais: "firmeza, ductilidade, fluidez" (SOLA-MORALES, 2002, p. 127). Aqui, tendo em vista o foco do presente trabalho, trataremos apenas da fluidez.

O conceito de fluido refere-se ao estado físico de gases e líquidos, à sua capacidade de deformação com a ação de forças externas e de modificação, ao admitir novas formas. Bauman (2001) apropria-se desse conceito e associa os fluidos às "ideias de leveza, mobilidade e inconstância" da sociedade contemporânea, fazendo metáforas entre a modernidade e o estado líquido (SQUAIELLA; MARCHELLI; IBIAPINA, 2015). Em Arquitetura, O conceito de liquidez é atribuído à possibilidade que o espaço construído tem de se adaptar às eventualidades; ao dinamismo no uso do espaço e do tempo; à capacidade de adaptação a atividades e à sustentabilidade (ROCHA, 2015). E, apesar de concebido como estável, estático e contínuo, esse espaço deve ser capaz de, fisicamente, receber qualquer tipo de troca (SOLA-MORALES, 2002, p. 134). A questão da fluidez pode ser abordada no processo de projeto e de análise gráfica, por meio de elementos como estrutura, programa e paisagem, por remeterem à obra e serem passíveis de exame (SQUAIELLA; MARCHELLI; IBIAPINA, 2015).

Quanto à criação de espaços fluidos, a estrutura é o elemento arquitetônico que auxilia nessa tarefa; o programa, por sua vez, influencia a concepção do 
projeto; e a paisagem pode ser analisada por meio da integração entre espaços internos e externos (SQUAIELLA; MARCHELLI; IBIAPINA, 2015). Assim, a concepção do espaço deve ser mais flexível e prever mudanças no programa, visto que a tecnologia digital (tecnologia móvel e internet) possibilita a ampliação e diversificação das atividades em um mesmo espaço. Essa capacidade modifica a condição de espaços físicos para a de espaços de fluxos, adaptáveis e aptos a permitir a comunicação.

O repertório tecnológico dos tempos atuais interfere no processo projetual e nas respostas espaciais, porém os elementos em relação aos quais é preciso tomar decisões projetuais determinantes são os mesmos, como: implantação no terreno, relações com o local e entorno, insolação, organização funcional, circulação, sistema estrutural e resultado formal/estético (ROCHA JR., 2014). Também se deve buscar novas formas de expressão gráfica para novos elementos, processos, materiais, assim como novos profissionais de design e da construção, entre outros (TASHEVA, 2012).

\subsection{Processo de projeto na contemporaneidade}

As propostas arquitetônicas contemporâneas, associadas à dinâmica do espaço, requerem "procedimentos de criação" e representação próprios, considerando que os métodos tradicionais já não são suficientes para atender às novas demandas (ROCHA, 2014). Nesse sentido, as tendências à integração social e ao rompimento do espaço homogêneo e com função definida, são diretrizes do processo projetual, que necessita de apoio (ZAERA-POLO, 2016).

Nos novos contextos sociais, culturais e econômicos, os desafios para o projeto passaram a ser maiores e mais complexos, porque, com o advento da globalização, o mercado passou a ser mais competitivo e a necessitar de maior produtividade e qualidade. Essas novas dinâmicas são acompanhadas de incertezas e riscos. Nesse contexto de mudanças rápidas, o estudo e a adoção de uma estrutura sistemática sobre o processo de projeto em arquitetura podem contribuir para melhorar, desenvolver e tornar mais eficiente a estratégia projetual na busca por respostas mais adequadas a problemas específicos em diferentes situações (PARSAEE; MOTEALLEH; PARVA, 2016), mesmo não havendo métodos rígidos ou universais entre os profissionais de prática de projeto (GOMES; PETRECHE, 2006).

Em arquitetura, o processo de projeto abrange questões relacionadas à compreensão dos problemas, à criatividade na busca de soluções para eles e ao conhecimento científico para os momentos de tomada de decisão, a fim de que esta se dê de maneira inteligente, competitiva e com respeito ao meio ambiente (KOWALTOWSKI; BIANCHI; PETRECHE, 2011). Além disso, é preciso levar em consideração que os problemas em design e arquitetura são abertos, admitem várias possíveis soluções e podem atender a critérios ou restrições em diferentes escalas.

Por outro lado, a criatividade em projeto implica também arriscar-se na síntese de uma solução (BONNARDEL; DIDIER, 2016). Para Crilly (2015), o processo de projeto criativo envolve a geração de novas ideias e está relacionado à qualidade da solução do projeto final. Nas fases iniciais de concepção, a geração de ideias é relevante para viabilizar qualidade e custos satisfatórios de execução do projeto final. 
Com base nas afirmações acima e com o intuito de fundamentar o tema desta pesquisa, será apresentado a seguir um estudo de apoio à solução de problema de projeto [problem solving], por meio de aplicação da linguagem gráfica à representação de conceitos de circulação e apoio às decisões criativas no processo de projeto.

\section{METODOLOGIA}

Adotou-se procedimento de pesquisa exploratória qualitativa e propositiva para análise da circulação (com método próprio) nos projetos selecionados de obras de uso público construídas, com tipologia de biblioteca e com informações técnicas disponíveis, com objetivo de levantar parâmetros, conceitos e configurações do espaço do ponto de vista da circulação de pessoas e de bens no espaço físico arquitetônico contemporâneo.

Trata-se de uma pesquisa voltada para a área de prática projetual, fundamentada nos conceitos de: resolução criativa de problemas na arquitetura; representação gráfica; e processo de projeto e da circulação (fluxo) de pessoas no projeto de arquitetura, a partir dos métodos de análise gráfica, conforme Figura 1. O método inclui: revisão bibliográfica; organização gráfica dos elementos da circulação (Quadro 1); seleção de amostra de projetos premiados para sua análise gráfica; redesenho e análise gráfica deles com base em legenda estruturada do Quadro 1; análise gráfica da amostra; análise dos resultados; e destaque de novos elementos para a questão da circulação em arquitetura.

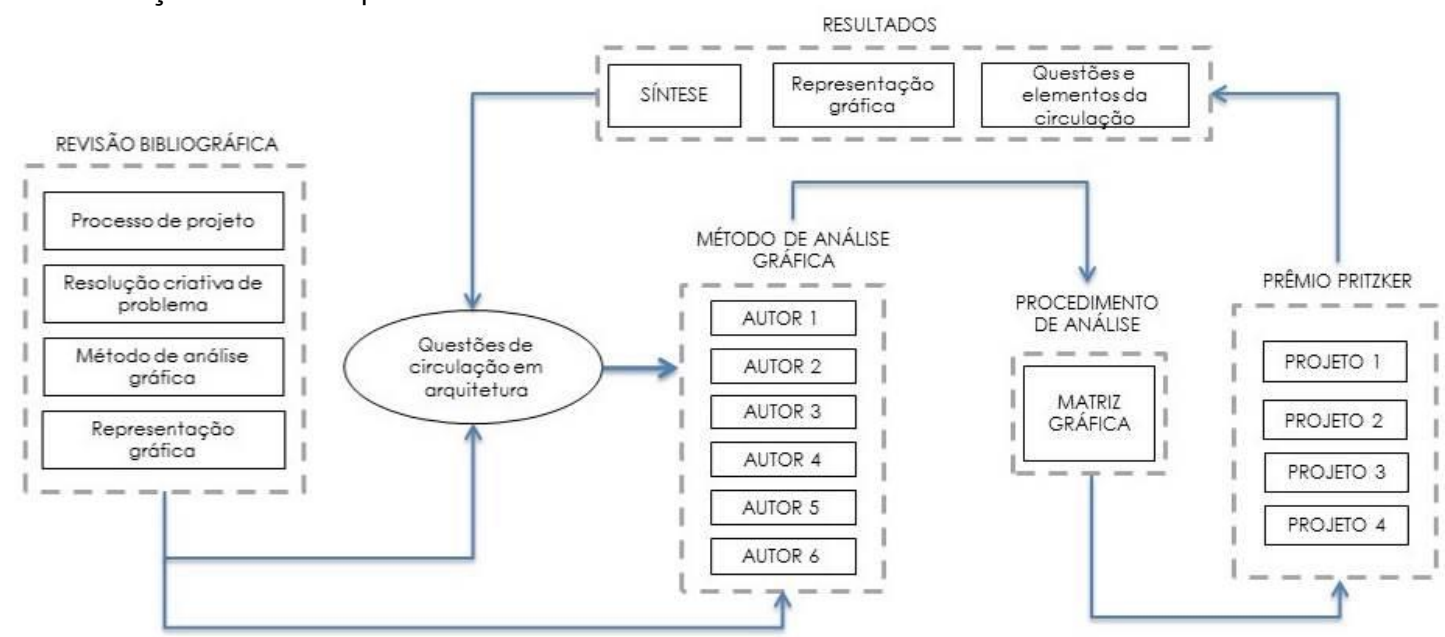

Figura1 - Estrutura dos procedimentos da pesquisa. Fonte: Victorio (2019).

A Fundamentação conceitual sobre as questões de circulação de pessoas e de bens na arquitetura partiu de bases teóricas e principalmente do modelo de análise gráfica de seis autores de referência - Baker (1998); Clark e Pause (1996); Ching (2008); Peña e Parshall (2012); Unwin (1997; 2013); e Radford et al. (2014) - e permitiu a estruturação, sistematização e representação, em forma de matriz gráfica, dos elementos e aspectos relacionados à circulação (Quadrol). Esses conceitos foram utilizados para procedimento de análise de quatro projetos desenvolvidos por arquitetos laureados com o prêmio Pritzker: Rem Koolhaas (Seattle Central Library - Washington, USA), Jacques Herzog e 
Pierre de Meuron (IKMZ BTU Cottbus - Cottbus, Germany); Toyo Ito (Sendai Mediatheque - Sendai, Japan), RCR Arquitectes - Rafael Aranda, Carme Pigem e Ramon Vilalta (De Krook Library - Ghent, Belgium), entre 2000 e 2010.

\section{RESULTADOS}

Criou-se uma matriz gráfica (Quadro 1), como resultado da estruturação, sistematização e representação gráfica dos aspectos de circulação, agrupados por itens conceituais (organização, orientabilidade [Wayfinding], funcionalidade, hierarquização, implantação e elementos compostos) sobre o fluxo de pessoas e bens na arquitetura.

O método de análise incluiu o redesenho de quatro projetos arquitetônicos de autoria dos arquitetos Rem Koolhaas, Herzog \& De Meuron, Toyo Ito e RCR Arquitectes -, resultando em desenhos que, em conjunto com 0 desenvolvimento de vistas isométricas explodidas e da codificação de elementos com códigos gráficos de representação, em forma de legenda, compuseram a base para o estudo e a interpretação da arquitetura deles com foco nos fluxos de peças (conforme exemplos da Figura 2). Essas análises resultaram no levantamento de novos elementos e aspectos da circulação, os quais, estruturados no Quadro 2, foram acrescentados ao acervo da literatura - e estão com destaque cinza no Quadro 1. 
Quadro 1 - Matriz gráfica parcial de conceitos de circulação, criada a partir de revisão bibliográfica e análise de projetos

\begin{tabular}{|c|c|c|c|c|c|c|c|c|c|}
\hline TEMA & $\begin{array}{c}\text { CONCEIT } \\
0\end{array}$ & \multicolumn{2}{|c|}{$\begin{array}{l}\text { ELEMENTOS DE } \\
\text { ANÁLISE }\end{array}$} & \multicolumn{6}{|c|}{ DETALHAMENTO } \\
\hline \multirow{23}{*}{$\begin{array}{l}\text { C } \\
\text { I } \\
\text { R } \\
\text { C } \\
\text { U } \\
\text { L } \\
\text { A } \\
\text { Ç } \\
\tilde{A} \\
\text { O }\end{array}$} & \multirow{4}{*}{\multicolumn{2}{|c|}{ 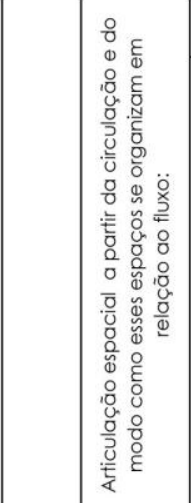 }} & \begin{tabular}{|l} 
Defini- \\
ção
\end{tabular} & $\begin{array}{l}\text { A análise dos diferen } \\
\text { de configuração esp }\end{array}$ & $\begin{array}{l}\text { thes tipos de circulaçã } \\
\text { pacial do projeto. }\end{array}$ & o e a maneira como & interagem com os es & spaços úteis, permite & entender $\mathrm{C}$ \\
\hline & & & \multirow[b]{3}{*}{ 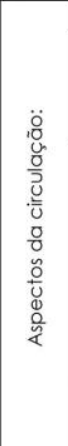 } & CENTRALIZADA & UNEAR & RADIAL & AGRUPADA & EM MALHA & COMPOSTA \\
\hline & & & & $\underbrace{1}_{\pi})_{\pi}^{K}$ & & & & & \\
\hline & & & & $\begin{array}{c}\text { Apresenta forma } \\
\text { não direcional. } \\
\text { acesso e entrada } \\
\text { são definidos pelas } \\
\text { características do } \\
\text { terreno ou pela } \\
\text { conexão a um } \\
\text { espaço secundário. }\end{array}$ & $\begin{array}{l}\text { Apresenta forma de } \\
\text { espaço serial, } \\
\text { relacionado } \\
\text { diretamente a um } \\
\text { outro espaço ou } \\
\text { conectados através } \\
\text { de um espaço } \\
\text { linear } \\
\text { independente. Sua } \\
\text { forma é flexivel e } \\
\text { adaptável ao } \\
\text { terreno. }\end{array}$ & $\begin{array}{c}\text { Caracteriza-se por } \\
\text { um espaço central } \\
\text { dominante e as } \\
\text { demais } \\
\text { organizações } \\
\text { lineares } \\
\text { configuradas de } \\
\text { maneira radial }\end{array}$ & $\begin{array}{l}\text { Caracteriza-se pela } \\
\text { proximidade fisica } \\
\text { de seus espaços }\end{array}$ & \begin{tabular}{|c|} 
Caracteriza-se \\
pelas formas e \\
espaços regulados \\
entre si por um \\
padrão em malha \\
tridimensional
\end{tabular} & $\begin{array}{l}\text { Caracteriza-se por } \\
\text { um espaço que } \\
\text { potencializa o } \\
\text { deslocamento com } \\
\text { menores condições } \\
\text { de controle por } \\
\text { causa do maior } \\
\text { número de } \\
\text { conexões (no } \\
\text { mínimo } 2 \text { conexões). }\end{array}$ \\
\hline & \multirow{11}{*}{ 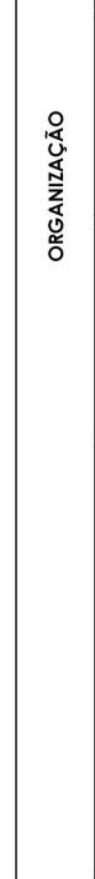 } & \multirow{3}{*}{ 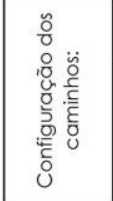 } & \begin{tabular}{|l} 
Defini- \\
ção
\end{tabular} & \multicolumn{6}{|c|}{$\begin{array}{l}\text { Os caminhos são definidos por um ponto de origem, uma sequência de espaços até o destino. A configuração do caminho } \\
\text { influencia e é influenciada pelo padrão de organização dos espaços que conecta. }\end{array}$} \\
\hline & & & \multirow[b]{2}{*}{ 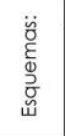 } & LINEAR & RADIAL & ESPIRAL & REDE & EIXO & COMPOSTO \\
\hline & & & & 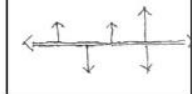 & & & & $\frac{1+11 T^{-}}{0.1 \frac{1}{0}+\frac{1}{6}}=$ & \\
\hline & & \multirow{3}{*}{ 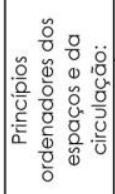 } & \begin{tabular}{|l} 
Defini- \\
ção
\end{tabular} & \multicolumn{6}{|c|}{$\begin{array}{l}\text { Os princípios de ordem são considerados recursos visuais capazes de manter, conceitualmente, um todo ordenado, unido e } \\
\text { harmonioso, composto por formas e espaços variados. }\end{array}$} \\
\hline & & & \multirow[b]{2}{*}{$\begin{array}{l}\ddot{\varepsilon} \\
\ddot{0} \\
\ddot{0}\end{array}$} & EIXO & HIERARQUIA & RITMO & SIMETRIA & REFERÊNCIA & TRANSFORMAÇĀO \\
\hline & & & & $\leftarrow=-=\cdot=1$ & $\underset{+1}{t}$ & $02 \pi$ & $\frac{\sqrt{2}}{2}-\frac{L}{r} \cdot \rightarrow$ & 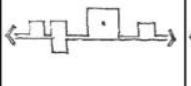 & \\
\hline & & \multirow{5}{*}{ 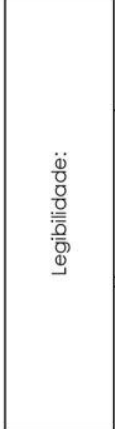 } & \begin{tabular}{|c} 
Defini- \\
ção
\end{tabular} & \multicolumn{6}{|c|}{$\begin{array}{l}\text { A circulação torna-se legível ao permitir o movimento ao longo dos percursos horizontais e verticais de forma segura, } \\
\text { confortável e livre de barreiras fisicas para se chegar ao destino. Deve ser clara, nítida, com diferenças nas dimensões, pisos, } \\
\text { paredes, cores, materiais e iluminação adequada. O deslocamento de pessoas e produtos em determinado espaço é } \\
\text { definido como fluxo, e está relacionado com a fluidez do movimento. }\end{array}$} \\
\hline & & & \multirow{2}{*}{ 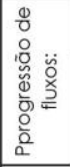 } & SEQUENCIAL & SEPARADO & MISTO & GRADIENTE ESPACIAL & $\begin{array}{l}\text { PERCURSO } \\
\text { ASCENDENTE }\end{array}$ & $\begin{array}{l}\text { FLUXO VERTICAL } \\
\text { CONTÍNUO }\end{array}$ \\
\hline & & & & 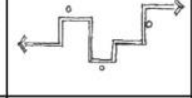 & $\underset{4}{\longrightarrow}$ & & $\equiv$ & & $\div+\frac{5}{\rightarrow}$ \\
\hline & & & \multirow[b]{2}{*}{ 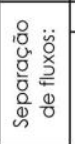 } & PEDESTRE & VEICULOS & BICICLEIAS & MERCADORIAS & SERVIÇOS & INFORMAÇÃO \\
\hline & & & & & & & & & \\
\hline & \multirow{8}{*}{ 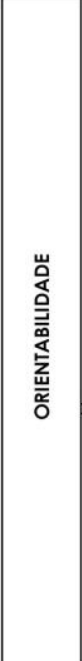 } & \multirow{5}{*}{ 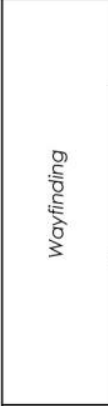 } & $\begin{array}{l}\text { Defini- } \\
\text { ção }\end{array}$ & \multicolumn{6}{|c|}{$\begin{array}{l}\text { "Wayfinding" trata das condições de orientabilidade no espaço construído e contribui para clareza na circulação e } \\
\text { legibilidade no percurso. Estuda os movimentos das pessoas e sua relação com o espaço. A linha de visão influencia o } \\
\text { percurso feito pelos usuários e o ângulo de aproximação afeta a legibilidade de entradas e saídas. }\end{array}$} \\
\hline & & & \multirow{2}{*}{ 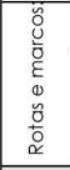 } & CONFIGURAÇĀO & REFERENCIAS & SINALIZAÇÃOO & VISTAS & ESCADA & $\begin{array}{l}\text { ARQUIBANCADA } \\
\text { URBANA }\end{array}$ \\
\hline & & & & $\sqrt{11}$ & & & & & \\
\hline & & & \multirow{2}{*}{ 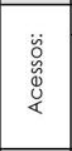 } & FRONTAL & OBULQUO & LATERAL & ACESSIVEL & HIERARQUIA & PASSEIO PÚBLICO \\
\hline & & & & $\bar{\uparrow}_{\uparrow}=$ & & $\vec{\Gamma}$ & Fin & 5 & +1 \\
\hline & & ڤ & \begin{tabular}{|c|c|c|c|} 
Defini- \\
ção
\end{tabular} & \multicolumn{6}{|c|}{$\begin{array}{l}\text { A permebilidade depende da relação entre circulação e espaço e, da maneira como se conectam. A adaptabilidade } \\
\text { depende da capacidade de se adequar a diferentes funções e alterar os requisitos espaciais ao longo do tempo. }\end{array}$} \\
\hline & & $\begin{array}{l}\frac{1}{0} \\
\frac{1}{0}\end{array}$ & $\begin{array}{l}0 \\
0 \\
0 \\
0\end{array}$ & $\begin{array}{l}\text { PERCORRE OS } \\
\text { ESPAÇOS }\end{array}$ & $\begin{array}{l}\text { ATRAVÉS DOS } \\
\text { ESPAÇOS }\end{array}$ & $\begin{array}{l}\text { TERMINA EM UM } \\
\text { ESPAÇO }\end{array}$ & ADAPTÁVEL & \begin{tabular}{|c|} 
PERCORRE AO REDOR \\
DO ESPAÇO
\end{tabular} & MULTIDIRECIONAL \\
\hline & & 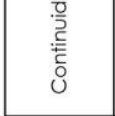 & 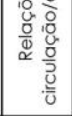 & & & & 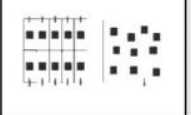 & & \\
\hline
\end{tabular}




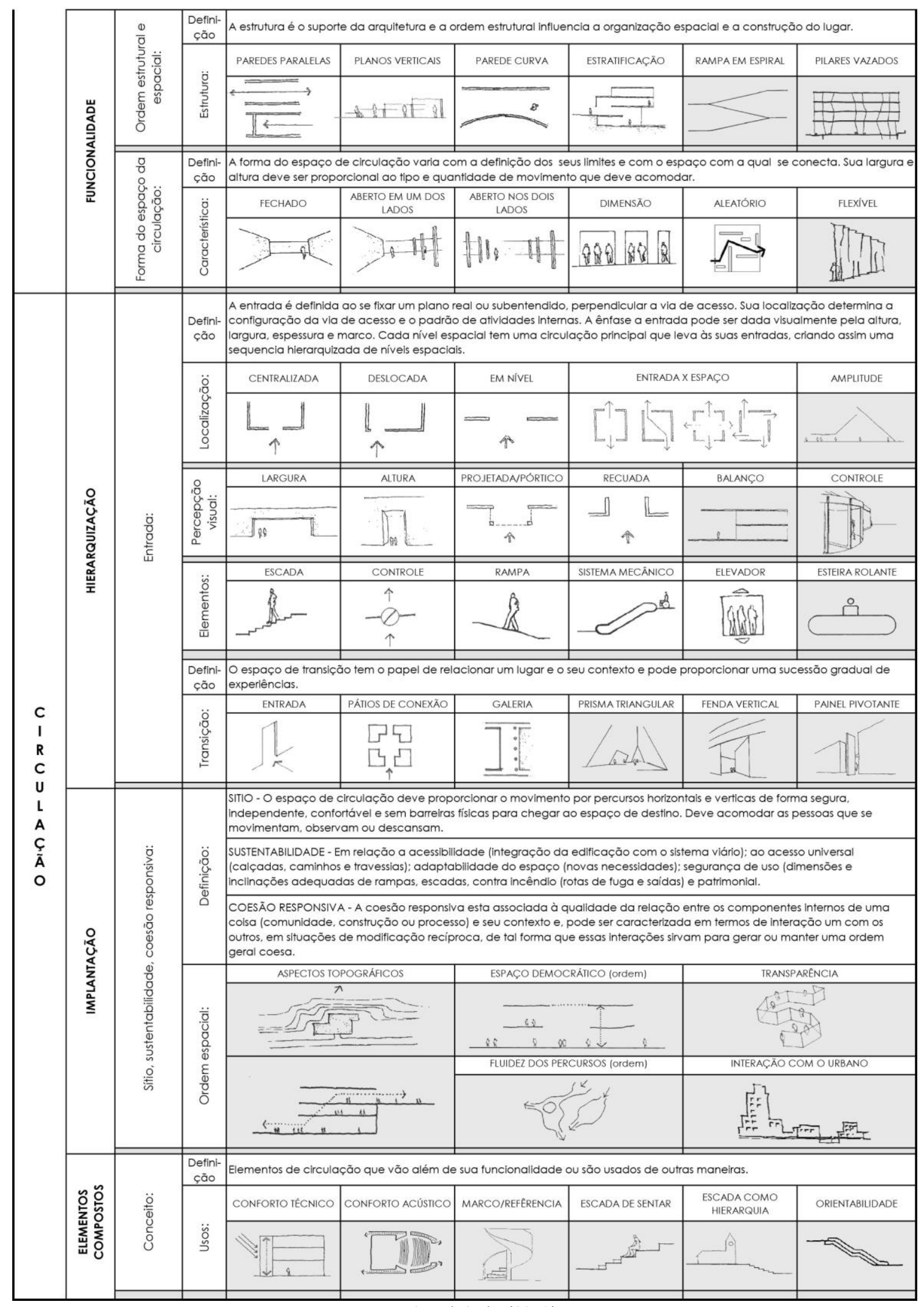

Fonte: Victorio (2019). 


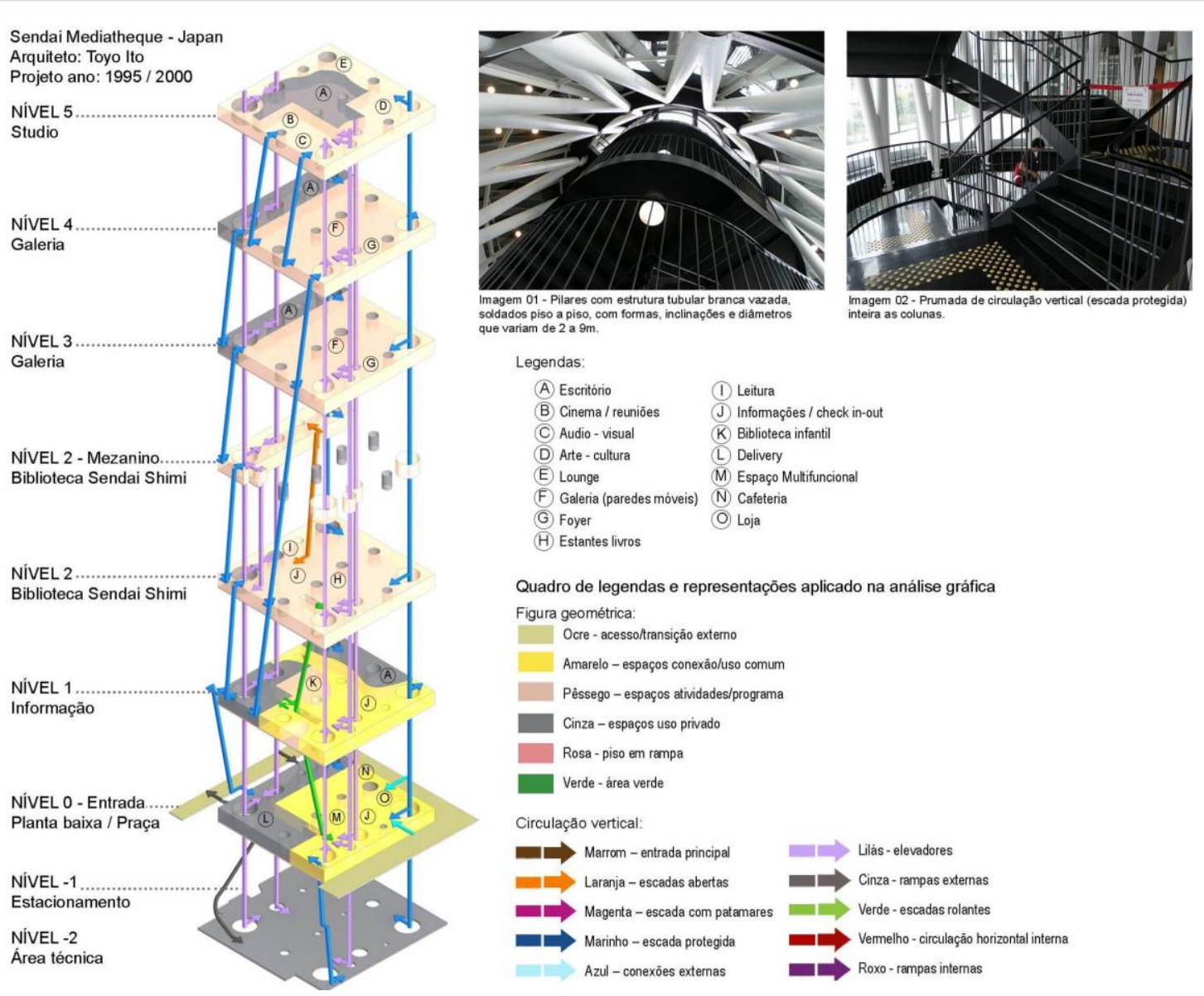

Figura 2 - Análise gráfica de projeto.

Fonte: Victorio (2019).

Os aspectos da circulação avaliados demostraram sua aplicação formal em soluções de projeto em relação a sítio/implantação; coesão responsiva/integração com o contexto urbano; organização espacial e funcional/setorização; orientação, identidade, movimento; acessibilidade, verticalização; transparência e fluidez (movimento). 
Quadro 2 - Representação gráfica das soluções de circulação em arquitetura

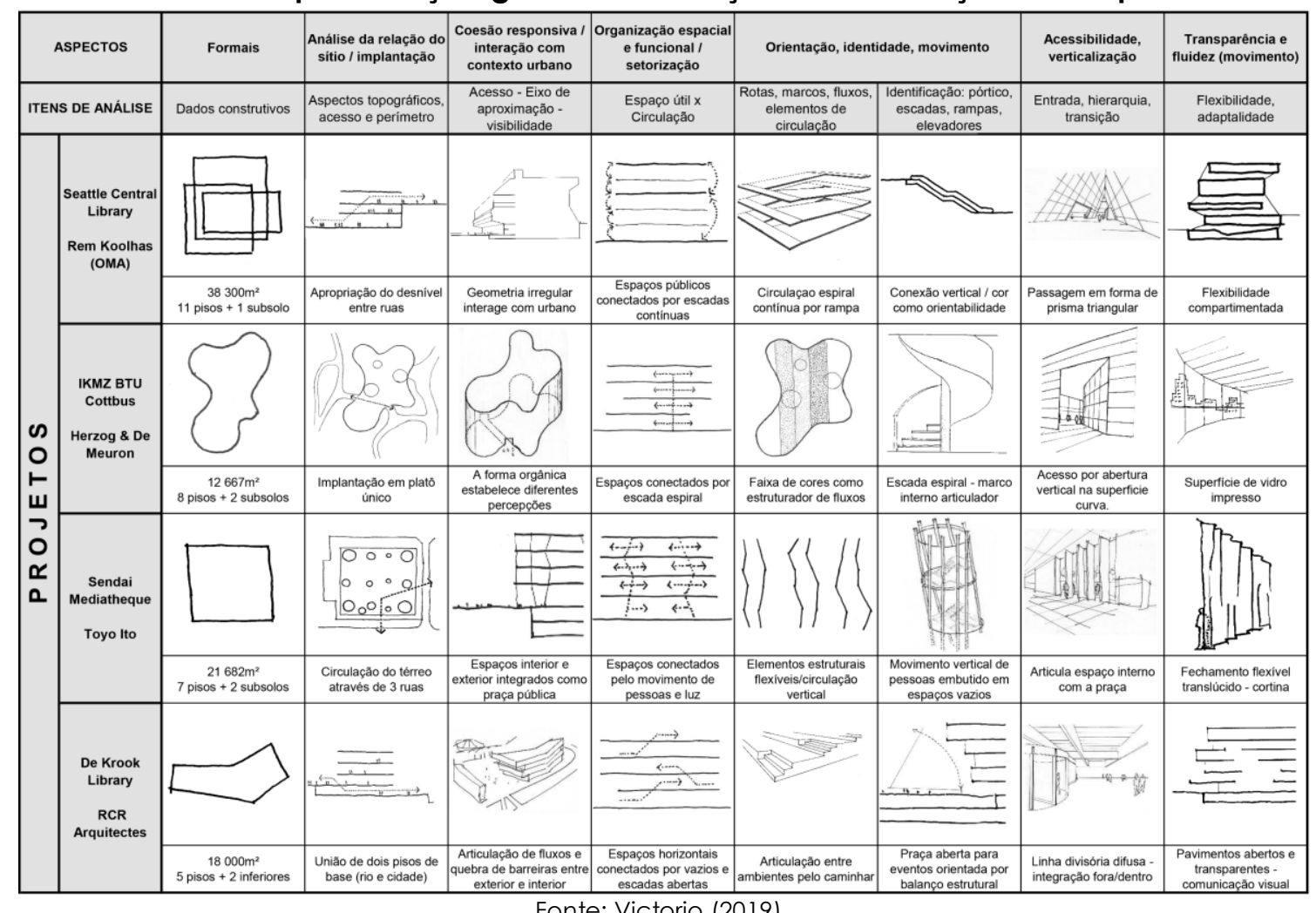

Fonte: Victorio (2019).

Essa análise demostrou-se eficiente para o levantamento de novos conceitos sobre circulação em arquitetura, além de estimular uma percepção visual sobre as soluções adotadas e estimular a geração de ideias na fase de síntese em um processo de projeto.

\section{CONCLUSÕES OU CONSIDERAÇÕES FINAIS}

O estudo alcançou seu objetivo principal de investigar o conceito de circulação na arquitetura, assim como permitiu a identificação de um conjunto de parâmetros e abordagens para a organização de elementos e soluções de problemas relacionados ao fluxo de pessoas e bens na arquitetura e para representá-los graficamente como suporte ao processo de projeto criativo.

Tais resultados demostram a importância dos percursos e da circulação já no processo de projeto, como apoio para a estruturação da complexidade das novas funções da arquitetura. Dessa maneira, esta pesquisa contribui, de forma específica, ao acrescentar novos aspectos e conceitos de circulação de pessoas e bens relacionados à arquitetura fluida. Ressalte-se que a tradução desses conceitos em linguagem gráfica é um importante recurso para o pensamento do projeto e de sua comunicação. Eles fazem parte do processo criativo e influenciam o pensamento e a resolução criativa de problemas, principalmente para arquitetos ainda com pouca experiência profissional.

O procedimento de análise gráfica mostrou-se eficaz ao revelar novos conceitos de circulação, criar uma linguagem de representação gráfica e 
facilitar a análise de projeto, permitindo que informações sejam avaliadas simultaneamente, além de estimular a geração de ideias. Assim, o método de análise gráfica ampliou a compreensão dos conceitos de circulação protagonistas dos projetos contemporâneos - e a percepção da estruturação espacial.

As questões de circulação são qualitativas e seus elementos impactam a estruturação, volumetria, funcionalidade e a inserção urbana de projetos. Diante disso, consideramos que o método de análise gráfica e os parâmetros levantados contribuem para a prática do processo de projeto profissional e acadêmico, permitindo resolução e configuração mais criativas do espaço arquitetônico.

Os resultados aqui apresentados devem, portanto, apoiar o desenvolvimento de métodos e ferramentas de design para apoiar esse processo criativo, tanto na prática profissional como no ambiente de ensino.

\section{REFERÊNCIAS}

BAKER, G. H. Analisis de la forma: urbanismo y arquitetura. 2. ed. Mexico: Gustavo Gilli, 1998.

BAUMAN, Z. Modernidade líquida. Rio de Janeiro: Zahar, 2001.

BONNARDEL, N.; DIDIER, J. Enhancing Creativity in the Educational Design Context: An Exploration of the Effects of Design Project-Oriented Methods on Students' Evocation Processes and Creative Output. Journal of Cognitive Education and Psychology Volume, v. 15, pp. 80-101, 2016.

CHING, F. D. K. Arquitetura: forma, espaço e ordem. 2 ed. São Paulo: Bookman, 2008.

CLARK, R. H.; PAUSE, M. Precedents in Architecture: Analytic Diagrams,

Formative Ideas, and Partis. Nova York: John Wiley \& Sons Inc., 1996.

CRILLY, N. Fixation and Creativity in Concept Development: The Attitudes and Practices of Expert Designers. Design Studies, v. 38, pp. 54-91, 2015.

DARKEN, R.P; PETERSON, B. Spatial Orientation, Wayfinding, and Representation. Handbook of Virtual Environment Technology. Stanney, K. Ed. 2001.

GOMES, V.; PETRECHE, J. R. D. Reflexão sobre metodologias de projeto arquitetônico. Ambiente Construído, Porto Alegre, v. 6, n. 2, pp. 7-19, abr./jun. 2006.

HUNTER, S. Architectural Wayfinding, M.Arch. IDeA Center, University at Buffalo, 2010. Disponivel em:

<http://www.udeworld.com/documents/designresources/pdfs/ArchitecturalW ayfinding.pdf>. Acesso em: 7 jun.2019.

KOWALTOWSKI, D. C. C. K.; BIANCHI, G.; PETRECHE, J. R. D. A criatividade no processo de projeto. In: KOWALTOWSKI, D. C. C. K. (org.). O processo de projeto em arquitetura: da teoria à tecnologia. São Paulo: Oficina de Textos, 2011, pp. 21-56.

NATAPOV, A.; DALTON, R. C.; HÖLSCHER, C. Building Circulation Typology and Space Syntax Predictive Measures. Proceedings of the 10th International Space Syntax Symposium, pp. 1-16, 2015. 
PEÑA, W. M.; PARSHALL, S. A. Problem Seeking. 4. ed. New York: John Wiley \& Sons, 2012.

PARSAEE, M.; MOTEALLEH, P.; PARVA, M. Interactive Architectural Approach (Interactive Architecture): An Effective and Adaptive Process for Architectural Design. HBRC .

RADFORD, A.; MORKOC ${ }_{y}$ S. B.; SELEN, B.; SRIVASTAVA, A. The Elements of Modern Architecture: Understanding Contemporary Buildings. 1. ed. London: Thames\&Hudson, 2014.

RADFORD, A. Responsive Cohesion in the Form Language of the Aalto Ateliers. Working papers - Alvar Alto Researchers' Network, pp. 1-10, 2012.Journal, v. 12, n. 3, pp. 327-336, 2016.

ROCHA, A. P. S. A-temporalidade do instantâneo: o tempo e o comportamento na arquitetura contemporânea. Dissertação (Mestrado em Arquitetura, Tecnologia e Cidade) - Universidade Estadual de Campinas (Unicamp), Campinas, 2015.

ROCHA JR., A. M. da. Domínio da forma: permanências e mutações nas composições arquitetônicas. Tese (Doutorado em Arquitetura e Urbanismo) Universidade Presbiteriana Mackenzie, São Paulo, 2014.

SOLA-MORALES, I. de. Territorios. Barcelona: Editoriao Gustavo Gili, 2002.

SQUAIELLA, R. B. F.; MARCHELLI, M. V.; IBIAPINA, B. G. Análise da Midiateca de Sendai como representação do espaço fluido. Projetar 2015, pp. 1-15, 2015.

TASHEVA, S. Semiotics of Architectural Graphics. Institute of Art Studies, Sofia, 2012.

TSCHUMI, B. Arquitetura e limites II. In: NESBITT, K. (ed.). Uma nova agenda para a arquietura: antologia teórica 1965-1995. 2. ed. São Paulo: Cosac Naify, 2008. UNWIN, S. Analysing Architecture. London: Routedge, 1997.

VAN DER VOORDT, T. J. M.; VAN WEGEN, H. B. R. Arquitetura sob o olhar do usuário. São Paulo: Oficina de Textos, 2013.

VIRILIO, P. O espaço crítico. São Paulo: Editora 34, 1993.

ZAERA-POLO, A. Arquitetura em diálogo: Alejandro Zaera-Polo. São Paulo: UBU Editora, 2016.

VICTORIO, E. R. As questões da circulação em arquitetura com base na análise de soluções de projetos contemporâneos. Dissertação (Mestrado em Arquitetura, Tecnologia e Cidade) - Universidade Estadual de Campinas (UNICAMP), Campinas, 2019. 